\title{
Ringmikrometerbeobachtungen von Kometen
}

am Refraktor (Objektivöffnung $18.6 \mathrm{~cm}$ ) der Düsseldorfer Sternwarte angestellt von Dr. W. Luther.

$\alpha$ app. $\quad|\log p \cdot A| \quad \delta$ app.

$|\log p \cdot d|$ Red. ad 1. app. $\mid$ *

I 910

\begin{tabular}{|c|c|c|c|c|}
\hline Jan. $\quad 27$ & 619 & 49 & +5 & 46.87 \\
\hline 29 & $5 \quad 56$ & 54 & - & 19.48 \\
\hline 29 & 626 & $4 \mathrm{I}$ & - & 2.80 \\
\hline 30 & 68 & 14 & -- & 46.89 \\
\hline $3 I$ & $6 \quad$ I 8 & 44 & -6 & 56.39 \\
\hline Febr. 9 & $6 \quad 37$ & I & +13 & 25.86 \\
\hline 13 & 626 & $5^{6}$ & -0 & 50.65 \\
\hline & 639 & 16 & & \\
\hline
\end{tabular}

I 9 IO

Halleyscher Komet $1909 \mathrm{c}$.

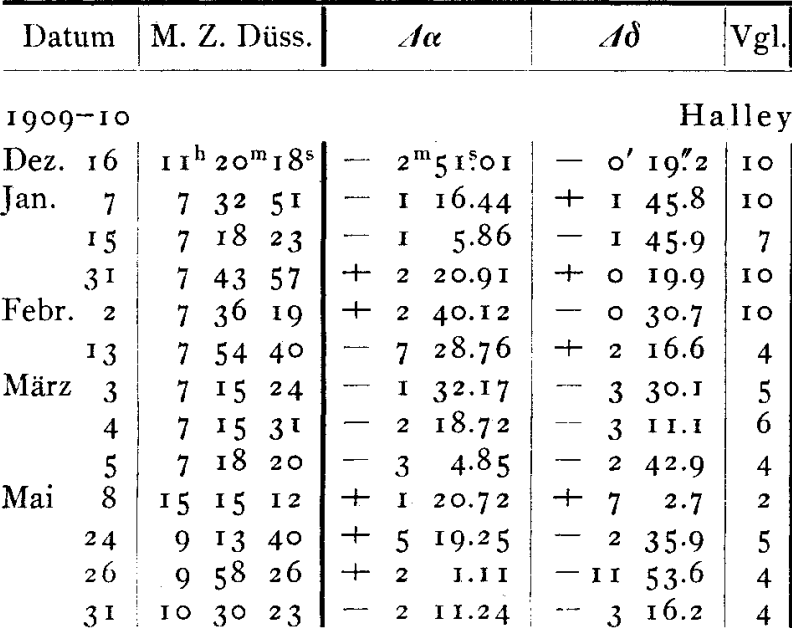

1909-10

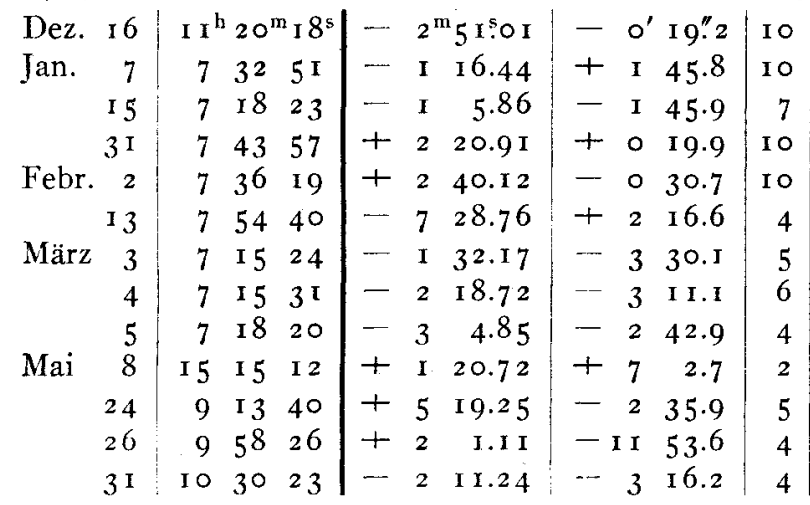

\begin{tabular}{l|rr|r}
0.733 & $+3^{\mathrm{s} .27}+\mathrm{r} 4 . \mathrm{I}$ & $\mathrm{I}$ \\
0.756 & $-0.58-1.8$ & 2 \\
0.768 & $-0.79-3.0$ & 4 \\
0.795 & $-\mathrm{I} .17-5.3$ & 5 \\
0.796 & $-1.2 \mathrm{I}-5.6$ & 6 \\
0.812 & $-\mathrm{I} .33-6.5$ & 7 \\
0.823 & $-\mathrm{I} .53-8.0$ & 8 \\
0.824 & $-1.53-8.1$ & 8 \\
0.825 & $-1.54-8.1$ & 8 \\
0.826 & $-0.85-6.8$ & 9 \\
0.811 & $-0.36+2.0$ & 10 \\
0.822 & $-0.16+0.6$ & 11 \\
0.831 & $+0.05-1.5$ & 12
\end{tabular}

Komet rgroa.

\begin{tabular}{|c|c|c|c|c|c|c|c|c|c|c|}
\hline & 32.0 & I & 21 & 24 & I 7.47 & $9 \cdot 560$ & - & I & 8 & 5.6 \\
\hline I 2 & 37.1 & 6 & 21 & 30 & 26 & $9.55^{\circ}$ & + & 0 & 47 & I. 4 \\
\hline 4 & I 8 . & 2 & 21 & 30 & 30.05 & 9.562 & + & 0 & 48 & 0.1 \\
\hline & 47.1 & 4 & 21 & 33 & 10.5 & $9.55^{6}$ & + & I & 37 & 20.6 \\
\hline & 6.7 & 5 & 21 & 35 & 41.9 & $9.50 \mathrm{I}$ & + & 2 & 23 & $5 \cdot 7$ \\
\hline & 34.2 & 2 & 21 & $5^{2}$ & $3^{6.23}$ & 9.570 & + & 7 & 12 & 28.3 \\
\hline & $31 \cdot 3$ & I & 21 & $5^{8}$ & $12 .^{\circ}$ & $9.57 \mathrm{I}$ & + & 8 & $4 \mathrm{I}$ & 33.9 \\
\hline & I 1.3 & 32 & 21 & $5^{8}$ & I 2.85 & $9.57 \mathrm{I}$ & + & 8 & $4 I$ & 28.1 \\
\hline
\end{tabular}

\begin{tabular}{l|ll|l}
0.835 & $-\mathrm{r} .92$ & $-\mathrm{I} 0.7$ & $\mathrm{r} 3$ \\
0.833 & $-\mathrm{I} .89$ & $-\mathrm{I} 0.7$ & $\mathrm{I} 4$ \\
0.833 & $-\mathrm{r} .88$ & $-\mathrm{I} 0.6$ & $\mathrm{I} 5$ \\
0.832 & $-\mathrm{r} .88$ & $-\mathrm{I} 0.6$ & $\mathrm{r} 6$ \\
$0.83 \mathrm{I}$ & $-\mathrm{I} .86$ & $-\mathrm{I} 0.6$ & $\mathrm{I} 7$ \\
$0.83 \mathrm{I}$ & $-\mathrm{I} .83-\mathrm{I} \mathrm{I} . \mathrm{I}$ & $\mathrm{I} 8$ \\
0.829 & $-\mathrm{I} .80$ & $-\mathrm{I} \mathrm{I} .0$ & $\mathrm{I} 9$ \\
0.832 & $-\mathrm{I} .80$ & $-\mathrm{I} \mathrm{I} .0$ & 20
\end{tabular}

Fayescher Komet rgroe.

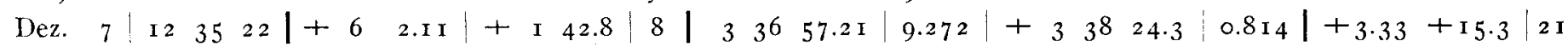

Mittlere Örter der Vergleichsterne.

\begin{tabular}{|c|c|c|c|c|}
\hline$*$ & $\alpha$ & & $\delta$ & Autorität \\
\hline & 1909.0 & 190 & 09.0 & \\
\hline I & $\begin{array}{c}3^{\mathrm{h}} 24^{\mathrm{m}} 2^{\mathrm{s}} \cdot 5^{8} \\
1910.0\end{array}$ & $\begin{array}{r}+14^{\circ} \\
191\end{array}$ & $\begin{array}{l}3^{\prime} 5^{\prime \prime} \cdot 2 \\
10.0\end{array}$ & AG Lpz I IоI 8 \\
\hline 2 & I $5^{8} \quad 3^{8.07}$ & +103 & $\begin{array}{ll}35 & 3.8\end{array}$ & $\begin{array}{l}9^{\mathrm{m}} 6 \text {, Düss. mikr. Anschluß } \\
\text { an } 3 \text {, Ep. I } 9 \text { I } 0.02\end{array}$ \\
\hline 3 & I $5^{8} \quad 9.34$ & +103 & $35 \quad 6.2$ & AG Lpz I 610 \\
\hline 4 & I $3^{6} .31 .16$ & +93 & $394 \mathrm{I} .2$ & $\mathrm{AG} \operatorname{Lp} z$ II $63^{2}$ \\
\hline 5 & $\begin{array}{lll}\text { I } & 2 & 0.23\end{array}$ & +82 & $23 \quad 9.6$ & $\gg \quad 40 \mathrm{I}$ \\
\hline 6 & $\circ 5^{8} \quad 45.73$ & $+8 \mathrm{I}$ & 1823.6 & $\gg \quad 379$ \\
\hline 7 & $\circ \quad 55 \quad 28.69$ & +75 & $56 \quad$ I 0.2 & $\begin{array}{l}1 / 2(\mathrm{AG} \mathrm{Lpz} \mathrm{II} 342+\mathrm{Kü} \\
\quad 397)\end{array}$ \\
\hline 8 & ○ $\quad 3342.89$ & +75 & $5^{8} 3^{2.1}$ & $\mathrm{AG} \mathrm{Lpz} \mathrm{II} 197$ \\
\hline 9 & 0127.72 & +94 & $44 \quad 40.3$ & AG Lpz II $6 \mathrm{I}$ \\
\hline
\end{tabular}

\begin{tabular}{|c|c|c|c|c|c|c|c|}
\hline$*$ & \multicolumn{3}{|c|}{$\alpha \mathrm{I} 9 \mathrm{I} 0.0$} & \multicolumn{3}{|c|}{$\delta 1910.0$} & Autorität \\
\hline IO & & $20^{n}$ & n 8.08 & + & $9^{\circ} 43^{\prime}$ & 3.' I & $\begin{array}{l}1 / 2(\text { AG Lpz II } 4572+\mathrm{Kü} \\
\quad 37 \text { I } 5)\end{array}$ \\
\hline I I & 9 & 2 & 43.59 & + & $64 I$ & 4.9 & $\mathrm{AG} \mathrm{Lpz} \mathrm{II} 4964$ \\
\hline I 2 & 9 & $5^{2}$ & 47.77 & + & 232 & $7 \cdot 7$ & AG Alb 39 I6 \\
\hline I 3 & $2 \mathrm{I}$ & 18 & $32 \cdot 52$ & - & I 9 & 26.9 & AG Nic 5439 \\
\hline I 4 & $2 \mathrm{I}$ & 30 & 48.25 & + & ○ 34 & 35.0 & $» \quad 5475$ \\
\hline I 5 & $2 I$ & 37 & 34.73 & + & $\circ 5^{2}$ & $29 . x$ & $\gg \quad 5497$ \\
\hline I 6 & $2 \mathrm{I}$ & 34 & $59 \cdot 32$ & + & I 50 & I 8.3 & AG Alb $75^{6} 5$ \\
\hline I 7 & $2 \mathrm{I}$ & 42 & 40.18 & + & 216 & 9.7 & $\geqslant \quad 7601$ \\
\hline I 8 & $2 \mathrm{I}$ & 39 & I 2.20 & + & 77 & $5 \cdot 2$ & AG Lpz II IO9 I \\
\hline I 9 & $2 \mathrm{I}$ & 59 & 5.16 & + & 838 & I $3 \cdot 7$ & $\gg \quad$ I IO9 I \\
\hline 20 & 22 & 0 & 45.60 & + & 844 & 50.5 & 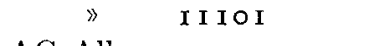 \\
\hline $2 \mathrm{I}$ & 3 & 30 & $5 \mathrm{I.77}$ & + & 336 & 26.3 & $\mathrm{AG} \mathrm{Alb} 103^{2}$ \\
\hline
\end{tabular}

Bemerkungen.

Halleyscher Komet.

I 909 Dez. I6. Den sternartigen Kern schätzte ich bei guter Luft I $^{\mathrm{m}} \cdot 5$. Die Nebelhülle ist außerordentlich zart und kaum wahrnehmbar, so daß sie die Gesamthelligkeit nicht merklich erhöht. Der Komet als Ganzes ist nahezu

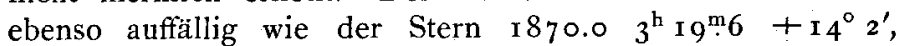
welcher auf der Pariser Karte roa die Größe ro ${ }^{\mathrm{m}}{ }_{5}$ hat. -
I910 Jan. 7. Die am Tage nach der Kometenbeobachtung ausgeführte Bestimmung des Vergleichsterns beruht auf ro Vergleichungen. Kern anscheinend nicht mehr ganz so sternartig wie Dez. r6. Nebelhülle sehr schwach. Gesamtauffälligkeit des Kometen $0^{\mathrm{m}} \cdot 5$ geringer als die des nicht in $\mathrm{BD}$ vorkommenden Vergleichsterns, den ich $9^{\mathrm{m}} \cdot 6$ schätze, und gleich der des Sterns $10^{\mathrm{m}} \cdot 5 \quad 185^{2.5} \mathrm{I}^{\mathrm{h}} 55^{\mathrm{m}} \cdot{ }^{\mathrm{O}}+10^{\circ} 23^{\prime} \mathrm{der}$ 
Pariser Karte 6. - Jan. 8. Um $6^{\text {h }} 5^{\text {m }}$ M. Z. Düss. war die Gesamtauffälligkeit des Kometen gleich der des Sterns ${ }^{\circ} 0^{\mathrm{m}} \cdot 3$ $185^{2.5} \mathrm{I}^{\mathrm{h}} 5^{\mathrm{m} .8}+10^{\circ} 7^{\prime}$ der Pariser Karte 6. - Jan. I 5. Es wurde trübe. Gesamtauffälligkeit gleich der des Sterns $9^{\mathrm{m}} \cdot 2$ I $855.0 \quad \mathrm{I}^{\mathrm{h}} 3 \mathrm{O}^{\mathrm{m}} 4^{\mathrm{I}} \cdot 5+9^{\circ} 3^{\prime} \cdot 3 \mathrm{BD}+9^{\circ} 198$. - Jan. 27. Bei schlechter Luft Komet nicht auffälliger als $9 \cdot 5$ I $\mathrm{I}^{\mathrm{h}} 8^{\mathrm{m}} \mathrm{I} 2 \mathrm{~s} \cdot 3$ $+8^{\circ}$ I6.。 BD + $8^{\circ}$ I98. - Jan. 3\%. Der Komet war im Sucher deutlich erkennbar und ebenso wie im Refraktor nahezu gleich auffällig wie die 4 Sterne: $9^{\mathrm{m}} \cdot 2 \mathbf{I}^{\mathrm{h}} \mathrm{o}^{\mathrm{m}} 45^{\mathrm{s}} \cdot \mathrm{o}$

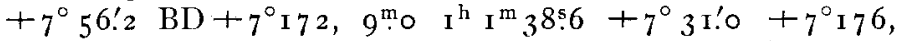
$9^{\mathrm{m}} \cdot 3 \mathrm{I}^{\mathrm{h}} \mathrm{I}^{\mathrm{m}} 44^{\mathrm{s}} \cdot 5+8^{\circ}{ }^{\circ} 5^{\prime} \cdot 7+8^{\circ} \mathrm{I} 79$ und $9^{\mathrm{m}} \cdot \mathrm{I}_{\mathrm{I}^{\mathrm{h}}} \mathrm{I}^{\mathrm{m}} 49^{\mathrm{s}} \cdot 9$ $+7^{\circ} 50^{\prime} 3+7^{\circ} 177$. Demnach war die Gesamtauffälligkeit $=9^{\mathrm{m}} \cdot \mathrm{I} 5$. Den sternartigen, von einer nahezu kreisrunden Nebelhülle umgebenen Kern schätzte ich $\mathrm{r}^{\mathrm{m}} \cdot 3$. Kein Schweifansatz. - Febr. 2. Heftiger Wind. Bilder meist gut. Im Refraktor schätzte ich die Gesamtauffälligkeit ähnlich der des Sterns I $855 . \circ\left(9^{\mathrm{m}} \mathrm{I}\right) \quad 0^{\mathrm{h}} 5^{6^{\mathrm{m}}} 23^{\mathrm{s}} \cdot 3+7^{\circ} 4^{2} \cdot 3 \mathrm{BD}+7^{\circ} \mathrm{I} 57$, im Sucher gleich der des Sterns $1855.0\left(8^{\mathrm{m}} .8\right)$ o o $^{\mathrm{h}} 55^{\mathrm{m}} 5^{2^{\mathrm{s}} .7}$ $+8^{\circ} 0^{\prime} 4 \mathrm{BD}+8^{\circ} 16$. . Febr. I3. Mondsichel im SW. Kern gleichhell wie $9^{\mathrm{m}} \cdot 5 \quad{ }^{1} 855.0 \quad \circ^{\mathrm{h}} 45^{\mathrm{m}} 14^{\mathrm{s}} \cdot 4+7^{\circ} 35^{\prime} \circ$ $\mathrm{BD}+7^{\circ} \mathrm{I}_{25}$. Gesamtauffälligkeit gleich der des Sterns $9^{\mathrm{m}}$ I I $855.00^{\mathrm{h}} 44^{\mathrm{ml}} 5^{\mathrm{s}} \cdot 2+7^{\circ} 3^{\mathrm{I}} \cdot 4 \mathrm{BD}+7^{\circ} \mathrm{I} 2 \mathrm{I}$. - Febr. I 4. Der Kern erschien um $7^{\text {h }}$ als winziges Pünktchen bei Mondschein, während die Koma relativ zum Kern deutlicher war. Der als äußerst blasser Nebel erscheinende Komet war gleich auffällig mit $9^{\mathrm{m}} \mathrm{I} \mathrm{BD}+7^{\circ} \mathrm{I}_{2}$. - März 2. Durch Vergleichung mit den Sternen $\mathrm{I} 855.0 \quad\left(8^{\mathrm{m}} . \mathrm{o}\right) \mathrm{o}^{\mathrm{h}} 30^{\mathrm{m}} 54^{\mathrm{s}} .7 \quad+7^{\circ} 4^{\circ} . \mathrm{I}$ $\mathrm{BD}+7^{\circ} 83$ u. 1 $855 . \circ\left(8^{\mathrm{m}} 8\right) \mathrm{o}^{\mathrm{h}} 3 \mathrm{o}^{\mathrm{m}} 5^{8 \mathrm{~s}} \cdot 2+7^{\circ} 28^{\prime} \cdot 3 \mathrm{BD}+7^{\circ} 8_{4}$ ergab sich die Gesamthelligkeit des Kometen zu $8^{\mathrm{m}} \cdot 4$. Es wurde gleich darauf um $7 \frac{1}{2^{h}}$ dunstig. - März 3. Der Komet erschien als blasser nebeliger Stern. Nach der fünften Vergleichung verschwand zuerst der Vergleichstern $9^{\mathrm{m}} \cdot \mathbf{I}$ I 855.0 o $30^{\mathrm{m}} 5^{2: 8}+7^{\circ} 39^{\prime} \cdot 9 \mathrm{BD}+7^{\circ} 82$ in den Dünsten des Horizonts, dann gleichzeitig der Komet und der Stern $8^{\mathrm{m}} .8$ I $855.00^{\mathrm{h}} 30^{\mathrm{m}} 5^{8 \mathrm{~s}} \cdot 2+7^{\circ} 28^{\prime} \cdot 3 \mathrm{BD}+7^{\circ} 84$, während $8^{\mathrm{m}} \cdot \mathrm{o}$ I $855.00^{\mathrm{h}} 30^{\mathrm{m}} 54^{\mathrm{s}} .7+7^{\circ}{ }_{4} 8^{\prime}$. $\mathrm{I}$ BD $+7^{\circ} 83$ noch deutlich sichtbar blieb. Gesamtauffälligkeit also $=8^{\mathrm{m}} 8$. - März 4. Luft gut. In der hellen Dämmerung hob sich der Komet nicht deutlicher vom Himmelsgrund $\mathrm{ab}$ als der Vergleichstern $9^{\mathrm{m}} \mathbf{I}$ $\mathrm{BD}+7^{\circ} 82$. Am Schlusse der Beobachtung erschien der Vergleichstern schwächer als der Komet. Gesamtauffälligkeit gleich der der Sterne 8.8 I $855.0 \quad 0^{\mathrm{h}} .28^{\mathrm{m}} 0^{\mathrm{s}} .7+7^{\circ} 2 \mathrm{I}^{\prime} \cdot 2$ $\mathrm{BD}+7^{\circ} 75$ und $8{ }^{\mathrm{m}} 8 \circ^{\mathrm{h}} 3 \mathrm{o}^{\mathrm{m}} 5^{8 \mathrm{~s}} \cdot 2+7^{\circ}{ }^{\circ} 8^{\prime} 3 \mathrm{BD}+7^{\circ} 84$. März 5. Komet war nahezu gleich hell wie $8^{\mathrm{m}} .8 \mathrm{BD}+7^{\circ} 75$ und verschwand bald. - April 22. Um $15^{\mathrm{h}} 50^{\mathrm{m}}$ M. Z. Düss. sah ich den Kometen trotz hellen Mondscheins im Refraktor deutlich, aber ohne Schweif. Er war im Sucher soeben sichtbar. Wegen der Dämmerung war im Refraktor der Stern $7^{\mathrm{m}}$. $\mathrm{BD}+7^{\circ} 5_{\mathrm{I}} \mathrm{OI}$ unsichtbar. Um $6^{\mathrm{h}} 6^{\mathrm{m}} \mathrm{M} . \mathrm{Z}$. Düss. war der Stern $\mathrm{BD}+7^{\circ} 5$ I 2 I ( $c$ Piscium Potsdam GW $6^{\mathrm{m}} .00$ ) im Refraktor noch deutlich sichtbar, im Sucher nur soeben zu ahnen. Um $16^{\mathrm{h}} 17^{\mathrm{m}} \mathrm{M}$. Z. Düss. war der Komet im Refraktor noch soeben deutlich zu erkennen, verschwand aber dann hinter einer Wolke. Die Gesamtauffälligkeit des Kometen schätze ich daher auf rund $6^{\mathrm{m}}$. - April 27. Bei dunstiger Luft und Mondschein war um $16^{\mathrm{h}} 9^{\mathrm{m}}$ M. Z. Düss. der Komet im Refraktor unsichtbar, während $\omega$ Piscium (Potsdam W $\left.4^{\mathrm{m}} \cdot 29\right)$ deutlich sichtbar war. - Mai 5 . Um $15^{\mathrm{h}} 23^{\mathrm{m}}$ m. Z. Düss. war der Komet bei dunstiger Luft im Refraktor als äußerst blasser runder Nebel soeben zu erkennen, während um $15^{\mathrm{h}} 3 \mathrm{O}^{\mathrm{m}} \omega$ Piscium $\left(4^{\mathrm{m}} \cdot 29\right)$ sehr hell, etwa $2^{\mathrm{m}}$ heller als vorher der Komet erschien. Gesamtauffälligkeit also kaum $6^{\mathrm{m}}$. - Mai 6. Um I $5^{\mathrm{h}} \mathrm{I}^{\mathrm{m}} \mathrm{m}$. Z. Düss. sah ich den Kometen in einer Wolkenlücke eine Minute lang als Scheibe von 0.5 Durchmesser ohne Schweif, und schätzte seine Helligkeit im Vergleich $\mathrm{zu}$ dem vorher eingestellten Stern 77 Pegasi (Potsdam $\mathrm{G} 5^{\mathrm{m}} \cdot 2$ ) etwa $4^{\mathrm{m}}$. - Mai 7 . Bei durchsichtiger Luft war der Komet deutlich sichtbar. Um $15^{\mathrm{h}}, 3^{\mathrm{m}} \mathrm{m}$. Z. Düss. nahm ich auf der Ostseite vom Positionswinkel $0^{\circ}$ durch $90^{\circ}$ bis $180^{\circ}$ eine fächerförmige Ausstrahlung wahr. Der Kern des Kometen war wesentlich schwächer als $\gamma$ Pegasi (W $3^{\mathrm{m}} \cdot 28$ Potsdam), aber seine Gesamtauffälligkeit $\operatorname{lag} \mathbf{I}_{5^{\mathrm{h}}} \mathbf{I}^{\mathbf{m}^{\mathrm{m}}}$ M. Z. Düss. zwischen der von $\omega$ Piscium ( $W_{4} 4^{\mathrm{m}} \cdot 29$ Potsdam) und $\gamma$ Pegasi $\left(\mathrm{W}_{3}^{\mathrm{m}} \mathbf{2}^{2}\right)$ näher der des letzteren, war also sehr nahe $3^{\mathrm{m}} 6$. Um ${ }^{\mathrm{h}} 27^{\mathrm{m}} \mathrm{M}$. Z. Düss. schätzte ich im Sucher den Kometen $0^{\mathrm{m}} \cdot 4$ heller als den vorher eingestellten $\omega$ Piscium $\left(4^{\mathrm{m}} 29\right)$. Um ${ }^{\mathrm{I}} 5^{\mathrm{h}} 3^{2^{\mathrm{m}}} \mathrm{M}$. Z. Düss. schätzte ich im Sucher und Refraktor $\gamma$ Pegasi ${ }^{m} \cdot 6$ heller als den Kometen. Um $15^{\mathrm{h}} 5^{2^{\mathrm{m}}}$ M. Z. Düss. war der Komet im Refraktor nur noch soeben zu ahnen, während $\gamma$ Pegasi noch ganz deutlich sichtbar war. Aus diesen Beobachtungen folgt demnach Gesamtauffälligkeit $=3^{m} .8 \mathrm{im}$ Vergleich zu zwei weißen Sternen. - Mai 8. Während der beiden Vergleichungen war die Luft gut; nachher trat Bewölkung ein. Der Kern des Kometen war nahezu gleich hell wie der mit ihm gleichzeitig im Gesichtsfeld befindliche Vergleichstern i 8, Potsdam RG $6^{\mathrm{m}} 60$, welcher in AG Lpz orange geschätzt ist. Die Gesant auffälligkeit des Kometen verglichen mit dem Vergleichstern $\left(6^{\mathrm{m}} 6\right)$ war nur $5^{\mathrm{m}} \cdot 6$. - Mai 18 . In der Nacht vom i 8 . zum I9. Mai, in der ein Einfluß des Kometenschweifes auf die Erdatmosphäre gemutmaßt wurde, fiel mir nur um $9^{\mathrm{h}} \cdot 5$ M. Z. Duiss. zwischen Mond und Zenit eine Anzahl auffallend, mindestens $10^{\circ}$ langer grauweißer Wogenwolkenstreifen von ca. $2^{\circ}$ Breite auf, welche, durch Zwischenräume getrennt, ziemlich genau von Norden nach Süden gerichtet waren. Mai 22. Um $8^{\mathrm{h}} 4^{\mathrm{I}^{\mathrm{m}}}$ M. Z. Düss. erschien der Komet im Sucher sternartig, im Refraktor als Nebelscheibchen von ca. $15^{\prime \prime}$ Durchmesser ohne Schweif. Um $9^{\text {h }}$ sah ich ihn in einem Opernglase als nebeligen Stern schwächer als Mars, Castor $\left(\mathrm{I}^{\mathrm{m}} \cdot 94\right)$ und Pollux $\left(\mathrm{I}^{\mathrm{m}} \cdot 5^{\mathrm{I}}\right)$ und erheblich schwächer als Procyon $\left(0^{\mathrm{m}} \cdot \mathrm{7}^{2}\right)$. Dagegen wird er heller gewesen sein als $\beta$ Can. min. $\left(3^{\mathrm{m}}, 20\right)$, den ich nicht sah. Gesamtauffälligkeit also rund $2^{\mathrm{m}}$. Mit bloßem Auge konnte ich ihn nur soeben erkennen. Er war für das bloße Auge kein auffälliges Objekt. Auch mehrere Besucher sahen keinen Schweif. Mai 23. Um $9^{\text {h }}$ M. Z. Düss. erschien der Komet in gegen Mai 22 veränderter Gestalt, nämlich am folgenden östlichen Rande halbkreisförmig begrenzt, am vorangehenden verlängert. Er hatte eine ausgesprochen ovale Form. Ausstrahlung nach Westen. - Mai 24. Der Komet sieht aus wie das übliche Kometenzeichen: West \& Ost. Die Mitte des Kerns ließ sich relativ gut auffassen. Bei Mondschein war weder im Sucher noch im Refraktor ein von der Sonne abgewandter Schweif zu sehen, aber der Komet hatte eine etwa $z^{\prime}$ lange nach Westen gerichtete fächerförmige Ausstrahlung zwischen den Positionswinkeln $190^{\circ}$ und $340^{\circ}$ nach meiner Schätzung, und zwar war die südlichere Hälfte dieses Lichtsektors $\left(\mathrm{PW}_{1} 90^{\circ}-240^{\circ}\right)$ wesentlich heller als die nörd- 
lichere. Durch Vergleichung mit $\beta$ Cancri (4988 Potsdam $\mathrm{G} 3^{\mathrm{m}} \cdot 74$ ) im Sucher schätzte ich die Gesamtauffälligkeit des Kometen gleich $2^{\mathrm{m}} \cdot 7$. Mit bloßem Auge sah ich Regulus deutlich, den Kometen nur sehr schwach. - Mai 26. Um $9^{\mathrm{h}} 9^{\mathrm{m}}$ M. Z. Düss. erschien im Sucher der Kern des Kometen entschieden $0^{\mathrm{m}} 6$ schwächer als $\zeta$ Hydrae (PD 5322 WG $\left.3^{\mathrm{m}} \cdot 34\right)$, der sogar auffälliger ist als der ganze Komet. Ich schätzte die Gesamtauffälligkeit gleich der von $\varepsilon$ Hydrae (PD $5252 \mathrm{WG}^{\mathrm{m}} \cdot 57$ ) und $\mathrm{I}^{\mathrm{m}} \cdot 2$ heller als $\varrho$ Hydrae (PD 5259 GW $\left.4{ }^{\mathrm{m}} \cdot 66\right)$. Der Kern war ${ }^{\mathrm{m}} \cdot 5$ heller als $\varrho$ Hydrae $\left(4^{\mathrm{m}} \cdot 66\right)$. Gesamtauffälligkeit etwa $2^{\text {In }}$ heller als $\omega$ Hydrae (PD 5407 G $55^{m}$ ). Kein Schweif. Ausstrahlung ähnlich wie Mai 24 . Radius der Nebelhiulle etwa ro'. Der in ruhigem Lichte leuchtende Kern bot Mai 26 einen unvergleichlich schönen Anblick dar. Er sah bei 50-f. Vergr. aus wie eine Perle aus lauterem Golde. Im Mittel Kern $=4^{\mathrm{m}} \circ$, Gesamtauffälligkeit $=3^{\mathrm{m}} \cdot 4$. - Mai $3 \mathrm{I}$. Kurze Schweifansätze nach $\mathrm{O}$ und SO, welche mehrere Besucher und ich in der hellen Dämmerung wahrzunehmen glaubten, waren nach einer Unterbrechung durch Wolken bei dunklerem Himmelsgrund nicht mehr sichtbar. Während der Beobachtung erschien der Kern nicht wie ein Stern, sondern mehr wie ein Nebelknoten. Sein Licht schien jedenfalls durch dichte Nebelschleier seiner Hülle hindurch. Die Mai 24 gesehene helle Ausstrahlung zur Sonne war nicht mehr sichtbar. Der Kern war nach allen Seiten von einer zarten Nebelhülle umgeben, die nach Schluß der hellen Dämmerung keinerlei Partien von besonders großer Intensität aufwies. $U m I I^{h} 2^{m}$ M. Z. Düss. schätzte ich die Gesamtauffälligkeit des Kometen gleich $4^{\mathrm{m}} \cdot 4$, nämlich $o^{\mathrm{m}} \cdot 3$ heller als die Helligkeit des mit dem Kometen im Gesichtsfeld des Suchers gleichzeitig sichtbaren Sterns I 5 Sextantis $=P D{ }_{588}$ I GW $4 \% 68$. Um I I $^{\mathrm{h}}$ I $_{4}^{\mathrm{m}}$ M. Z. Düss. war dieser Stern schon absolut unsichtbar, während ich den Kometen noch soeben deutlich erkennen konnte. Juni 9. Um $10^{\mathrm{h}}{ }_{2} 8^{\mathrm{m}}$ M. Z. Düss. war der Komet nur soeben als blasser Nebel zu ahnen, während der Stern $6{ }^{\mathrm{m}} \cdot 5$ BD $-0^{\circ} 233^{2}$ und $7^{\mathrm{m}} \cdot \mathrm{o} \mathrm{BD}-\mathrm{O}^{\circ} 234 \mathrm{I}$ noch ganz deutlich sichtbar waren.

Komet r9ioa.

I9 1o Jan. 27. Der Kern des Kometen erschien als scharf begrenzte kreisrunde Scheibe von ca. $20^{\prime \prime}$ Durchmesser. Die Achse des schmalen Schweifes lag nach Schätzung im Sucher im PW. $40^{\circ}$. Um $7^{\mathrm{h}} 8^{\mathrm{m}}$ M. Z. Düss. konnte ich zwar den Kern mit bloßem Auge nicht wahrnehmen, aber den vom Horizont ziemlich senkrecht aufsteigenden und in größerer Höhe nach Süden umbiegenden Schweif bis in die Gegend von $\gamma$ Piscium verfolgen. - Jan. 29. Der Komet ist schwächer als Jan. 27. Die beiden äußeren Randpartien des Schweifes waren erheblich heller als die mittleren Partien. Der Kern ist verschwommen wie Jan. 27. Seinen Durchmesser schätze ich auf $15^{\prime \prime}$. Den langen blassen Schweif konnte ich um $6^{\mathrm{h}} 5^{\mathrm{I}}$ M. Z. Düss. bis dicht an $\alpha$ Pegasi mit bloßem Auge verfolgen. - Jan. 30. Die Konturen des Kerns waren trotz befriedigender Luftbeschaffenheit undeutlicher als Jan. 27 . Mit bloßem Auge konnte ich den Schweif bis $\alpha$ Pegasi verfolgen, und sah auch den Kopf auf einen Augenblick. Mit dem Opernglase schätzte ich die Helligkeit des Kometen- kopfes gleich der von 2 Pegasi (PD I $2940 \mathrm{GW} 4^{\mathrm{m}} \cdot \mathrm{o} 8$ ). Im Sucher erschien der Kern $I^{\mathrm{m}} 5$ heller als der Vergleichstern $d$ Aquarii (PD I $\left.2566 \mathrm{G} 5^{\mathrm{m}} \cdot 26\right)$. Also im Mittel Komet $=3^{\mathrm{m}} \cdot 9$ ). - Jan. 3I. Um $5^{\text {h }} 5^{0^{m}}$ M. Z. Düss. schätzte ich im Sucher den Kometen $\mathrm{I}^{\mathrm{m}}$ heller als $d$ Aquarii $\left(5^{\mathrm{m}} \cdot 26\right)$. Um $6^{\mathrm{h}} \mathbf{3 2}^{\mathrm{m}}$ M. Z. Düss. schätzte ich im Opernglase bei durchsichtiger Luft den Kopf des Kometen gleich hell mit $\varepsilon$ Pegasi (PD I $\left.2628 \mathrm{G} 2^{\mathrm{m}} \cdot 76\right)$. Letztere Schätzung wurde bestätigt durch meine Frau, die im Garten den Kometen mit bloßem Auge betrachtete. Um $6^{\mathrm{h}} 43^{\mathrm{m}}$ M. Z. Düss. erschien der Kopf bei sehr tiefem Stande (Höhe $3^{\circ} \cdot 3$ ) im Refraktor nicht heller als der Vergleichstern I I Pegasi (PD I 266 I GW $5^{\mathrm{m}}$ 80). - Febr. 5. $\mathrm{Um} 6^{\mathrm{h}} 7^{\mathrm{m}}$ M. Z. Düss. erschien der Komet im Refraktor als runder Nebel ohne Schweif und verschwand bald darauf ziemlich gleichzeitig mit dem Stern 7 Pegasi (PD I 2600 G $5^{\mathrm{m}} \cdot 29$ ) in den Dïnsten des Horizonts. - Febr. 9. Deklination weniger sicher, da wegen der Dämmerung ein schlecht passender Vergleichstern gewählt werden mußte. Komet erschien als runder Nebel ohne Schweif mit einem Kern, den ich $0^{\mathrm{m}} \cdot 5$ schwächer schätzte als den Vergleichstern PD I $262 \mathrm{x}$ G $6^{\mathrm{m}} \cdot 6_{5}$, also $7^{\mathrm{m}} \cdot \mathbf{r}$. Die Gesamtauffälligkeit des Kometen war $0^{\mathrm{m}} .5$ heller als derselbe Stern, also $6^{\mathrm{m}} 2$. Die Deklination der ersten Beobachtung, welche nur auf einem Durchgang beruht, scheint unsicher zu sein. Um $6^{\text {h }}$ I $0^{\mathrm{m}}$ M. Z. Duiss. trat der Komet gleichzeitig mit dem Stern $8^{\mathrm{m}} \cdot 3 \mathrm{BD}+8^{\circ} 478_{2}$ aus der hellen Dämmerung heraus. Die Gesamtauffälligkeit war also $8^{\mathrm{m}} \cdot 3$. Um $7^{\mathrm{h}} \mathrm{M}$. Z. Düss. waren Komet und Sterne bereits im Dunst des Horizontes verschwunden. - April I I. Der Komet erschien nur als blasser Hauch. Gesamthelligkeit I I $^{\mathrm{m}} \cdot 5$.

Der Komet rgio a und der Halleysche Komet waren I910 Febr. I3 von annähernd gleicher Auffälligkeit. Der Komet rgro a stand aber bei der Beobachtung dem Horizont näher, und die Stellung zum Monde war ebenfalls verschieden.

\section{Fayescher Komet igioe. \\ Kern $10^{\mathrm{m}} \cdot 4$. Nebelhülle sehr zart.}

Die Beobachtungen sind nach der Auge- und OhrMethode angestellt, und bei der Ableitung der vorstehenden Differenzen $\&$ - * ist sowohl Eigenbewegung als Refraktion berücksichtigt worden.

$\mathrm{Da}$ ich bei meinen Planetenbeobachtungen mehrfach den Eindruck gehabt habe, daß das Planetenlicht in stärkerem Maße durch einsetzende geringere Durchsichtigkeit der Luft geschwächt werde als das Fixsternlicht, erscheint es mir sehr wohl möglich, daß Helligkeitsausbrüche bei Kometen, wie z. B. der von mir I9 Io Jan. 3 I bei Komet rg Io a beobachtete, zuweilen lediglich die Folge plötzlich eintretender »Kometenklarheit «, d. h. größerer Durchsichtigkeit der Atmosphäre resp. der Räume zwischen Komet und Erde sind. Daß außerdem reelle anormale sprungweise Vergrößerungen der Helligkeiten der Kometen statthaben werden, wenn deren Kopf von einem, einem Sonnenfleck entstammenden Energiestrahl getroffen wird, kann nicht nur als wahrscheinlich bezeichnet werden, sondern es dürfte diese Erscheinung einerseits und die Kometenklarheit andererseits vielleicht auf eine und dieselbe kosmische Ursache zurückzuführen sein.

Düsseldorf, I 9 I о Dez. iо. 